\title{
Boundedness of Stochastic Delay Differential Systems with Impulsive Control and Impulsive Disturbance
}

\author{
Liming Wang, ${ }^{1}$ Baoqing Yang, ${ }^{2}$ Xiaohua Ding, ${ }^{1}$ and Kai-Ning Wu${ }^{1}$ \\ ${ }^{1}$ Department of Mathematics, Harbin Institute of Technology at Weihai, Weihai 264209, China \\ ${ }^{2}$ Control and Simulation Center, Harbin Institute of Technology, Harbin 150001, China \\ Correspondence should be addressed to Kai-Ning Wu; kainingwu@163.com
}

Received 27 February 2014; Accepted 5 August 2014

Academic Editor: Weihai Zhang

Copyright (c) 2015 Liming Wang et al. This is an open access article distributed under the Creative Commons Attribution License, which permits unrestricted use, distribution, and reproduction in any medium, provided the original work is properly cited.

\begin{abstract}
This paper considers the $p$-moment boundedness of nonlinear impulsive stochastic delay differential systems (ISDDSs). Using the Lyapunov-Razumikhin method and stochastic analysis techniques, we obtain sufficient conditions which guarantee the $p$-moment boundedness of ISDDSs. Two cases are considered, one is that the stochastic delay differential system (SDDS) may not be bounded, and how an impulsive strategy should be taken to make the SDDS be bounded. The other is that the SDDS is bounded, and an impulsive disturbance appears in this SDDS, then what restrictions on the impulsive disturbance should be adopted to maintain the boundedness of the SDDS. Our results provide sufficient criteria for these two cases. At last, two examples are given to illustrate the correctness of our results.
\end{abstract}

\section{Introduction}

Boundedness is an important property of a given system; for example, in the population models, the boundedness of a biological population is strongly connected with the persistence and extinction [1]. Another important application is on the stability; the practical stability actually is of a kind of boundedness [2]. Impulsive phenomena widely exist in the real world, and known, impulsive effects can change the properties of a given system; for example, given an unstable system, if a suitable impulsive strategy, including the impulsive strength and impulsive moments, is adopted, this system can be stabilized [3]. It is easy to understand that the impulsive effects can destroy the boundedness of a given system when the impulsive strength is large enough and the impulsive interval is small enough. Time delay is extensive in the engineering and applications and impulsive delay differential systems were considered in lots of papers [3-9]. The boundedness of impulsive delay differential systems has also been paid considerable attentions in the past decades. In [10], the authors presented sufficient conditions for uniform ultimate boundedness by virtue of the Lyapunov functional method. The boundedness of variable impulsive perturbations system was considered in [11] and the eventual boundedness was studied in [12]. Recently, the perturbing Lyapunov function method was also used in the study of boundedness [13].

Stochastic noise is ubiquitous [14-16] and stochastic delay differential systems (SDDSs) have been one of the focuses of scientific research for many years. Many properties of SDDSs have been studied and lots of papers were published; see $[17,18]$ and the references therein. Being the wide existence of stochastic delay and impulsive effects, it is a natural task to consider the stochastic delay differential systems with impulsive effects. These systems are described by impulsive stochastic delay differential systems (ISDDSs). In the past ten years, the stability of ISDDSs has attracted a lot of researchers, and a great deal of results on the stability of ISDDSs have been reported; see [19-24] and the references therein.

However, little attention has been paid to the boundedness of ISDDSs. In this paper, the boundedness of ISDDSs is considered under two cases. The first case is that the SDDSs may be unbounded, then what kind of impulsive strategy should be taken to make the system be bounded. 
The second case is that the SDDSs are bounded, then this system can tolerate what kind of impulsive effect to maintain the boundedness.

In this paper, sufficient conditions are presented to guarantee the boundedness of ISDDSs; these conditions also admit the global existence of solutions for ISDDSs, which usually was a standard assumption in many papers [2527]. Making use of the Lyapunov-Razumikhin method, we generalize the results of [10] to the stochastic situation. At last, two examples are given to illustrate the correctness of our results.

\section{Preliminaries and Model Description}

Let $\left(\Omega, F,\left\{F_{t}\right\}_{t \geqslant 0}, P\right)$ be a complete probability space with a filtration $\left\{F_{t}\right\}_{t \geqslant 0}$ satisfying the usual conditions (i.e., the filtration contains all $P$-null sets and is right continuous). Let $\mathbb{R}=(-\infty,+\infty), \mathbb{R}^{+}=[0,+\infty)$, and $\mathbb{N}=\{1,2, \ldots\}$. If $A$ is a vector or a matrix, its transpose is denoted by $A^{T}$. Consider $P C\left(\mathbb{J} ; \mathbb{R}^{n}\right)=\left\{\varphi: \mathbb{l} \rightarrow \mathbb{R}^{n}, \varphi(s)\right.$ is continuous for all but at most countable points $s \in \mathbb{J}$ and at these points, $\varphi\left(s^{+}\right)$and $\varphi\left(s^{-}\right)$exist and $\left.\varphi\left(s^{+}\right)=\varphi(s)\right\}$, where $\rrbracket \subset \mathbb{R}$ is an interval and $\varphi\left(s^{+}\right)$and $\varphi\left(s^{-}\right)$denote the right-hand and left-hand limits of the function $\varphi(s)$ at time $s$, respectively. Consider $P C^{1,2}=\{\varphi(t, x): \varphi(\cdot, x) \epsilon$ $P C$ and $\varphi(t, x) \in C^{1,2}$ if $t$ is not at the uncontinuous points $s\}$. Let $P C_{F_{0}}^{b}\left([-\tau, 0] ; \mathbb{R}^{n}\right)\left(P C_{F_{t}}^{b}\left([-\tau, 0] ; \mathbb{R}^{n}\right)\right)$ denote the family of all bounded $F_{0}\left(F_{t}\right)$-measurable, $P C$-valued random variables. Let $|\cdot|$ be the Euclidean norm in $\mathbb{R}^{n}$ and $\|\varphi\|_{\tau}=$ $\sup _{-\tau \leqslant \theta \leqslant 0}|\varphi(t+\theta)|$.

Consider the following nonlinear impulsive stochastic delay differential system:

$$
\begin{gathered}
d x(t)=f\left(t, x_{t}\right) d t+g\left(t, x_{t}\right) d B(t), \\
t>t_{0}, \quad t \neq t_{k}, \quad k \in \mathbb{N}, \\
x\left(t_{k}\right)=x\left(t_{k}^{-}\right)+I\left(t_{k}, x\left(t_{k}^{-}\right)\right), \quad k \in \mathbb{N}, \\
x\left(t_{0}+s\right)=\varphi(s), \quad s \in[-\tau, 0],
\end{gathered}
$$

where $x_{t}(s)=x(t+s), s \in[-\tau, 0], f: \mathbb{R}^{+} \times P C\left([-\tau, 0], \mathbb{R}^{n}\right) \rightarrow$ $\mathbb{R}^{n}, g: \mathbb{R}^{+} \times P C\left([-\tau, 0], \mathbb{R}^{n}\right) \rightarrow \mathbb{R}^{n \times m}, I: \mathbb{R}^{+} \times \mathbb{R}^{n} \rightarrow \mathbb{R}^{n}$ and satisfies global Lipschitz condition, $\tau$ represents the delay in system (1), impulsive moment $t_{k}$ satisfies $0<t_{1}<t_{2}<\cdots<$ $t_{n}<\cdots$, and $t_{k} \rightarrow \infty$ as $k \rightarrow \infty$. $B(t)$ is an $m$-dimensenal Brownian motion and $\varphi(s) \in P C_{F_{0}}^{b}\left([-\tau, 0], \mathbb{R}^{n}\right)$.

Given a function $V \in P C^{1,2}: \mathbb{R}^{+} \times \mathbb{R}^{n} \rightarrow \mathbb{R}^{+}$, the operator $\mathscr{L}$ of $V(t, x)$ with respect to system (1) is defined by

$$
\begin{aligned}
\mathscr{L} V(t, x)= & V_{t}+V_{x} f\left(t, x_{t}\right) \\
& +\frac{1}{2} \operatorname{trace}\left[g^{T}\left(t, x_{t}\right) V_{x x} g\left(t, x_{t}\right)\right],
\end{aligned}
$$

where

$$
\begin{aligned}
V_{t} & =\frac{\partial V(t, x)}{\partial t}, \\
V_{x} & =\left(\frac{\partial V(t, x)}{\partial x_{1}}, \frac{\partial V(t, x)}{\partial x_{2}}, \ldots, \frac{\partial V(t, x)}{\partial x_{n}}\right)^{T}, \\
V_{x x} & =\left(\frac{\partial^{2} V(t, x)}{\partial x_{i} \partial x_{j}}\right)_{n \times n} .
\end{aligned}
$$

Definition 1. System (1) is said to be

(1) $p$-moment bounded if, for every $B_{1}>0$ and $t_{0} \epsilon$ $\mathbb{R}_{+}$, there exists $B_{2}=B_{2}\left(t_{0}, B_{1}\right)$ such that if $\varphi \in$ $P C_{F_{0}}^{b}\left([-\tau, 0], \mathbb{R}^{n}\right)$ with $E\|\varphi\|_{\tau}^{p} \leqslant B_{1}$ and $x=x\left(t, t_{0}, \varphi\right)$ is a solution of $(1)$, then $E\left|x\left(t, t_{0}, \varphi\right)\right|^{p} \leqslant B_{2}$ for all $t \geqslant t_{0}$

(2) $p$-moment uniformly bounded if the system (1) is $p$-moment bounded and $B_{2}$ is independent of $t_{0}$;

(3) $p$-moment ultimately bounded if the system (1) is $p$-moment bounded and there exists a positive constant $B$ such that for every $B_{3}>0$ and $t_{0} \in \mathbb{R}^{+}$there exists some $T=T\left(t_{0}, B_{3}\right)>0$; if $\varphi \in P C_{F_{0}}^{b}\left([-\tau, 0], \mathbb{R}^{n}\right)$ with $E\|\varphi\|_{\tau}^{p} \leqslant B_{3}$, then $E\left|x\left(t, t_{0}, \varphi\right)\right|^{p} \leqslant B$ for $t \geqslant t_{0}+T$;

(4) $p$-moment uniformly ultimately bounded, if the system (1) is $p$-moment ultimately bounded and $T$ is independent of $t_{0}$.

\section{Boundedness with Impulsive Control}

In this section, we consider the first case: when the given SDDS may not be bounded, we adopt an impulsive strategy to get the boundedness. The main result is stated as follows.

Theorem 2. Assume there exist a positive function $V(t, x) \in$ $P C^{1,2}$ and positive constants $\rho, p, a, b, \gamma, \lambda$, where $0<\lambda<1$ and $1-\lambda-\gamma \tau>0$, such that

(1) $a|x|^{p} \leqslant V(t, x) \leqslant b|x|^{p}$ for any $(t, x)$;

(2) for $t \neq t_{k}$, any $s \in[-\tau, 0]$, and $\phi(t) \in P C\left([-\tau, 0], \mathbb{R}^{n}\right)$, $\mathscr{L} V(t, \phi(0)) \leqslant \gamma V(t, \phi(0))$ whenever $V(t, \phi(0)) \geqslant$ $\lambda V(t+s, \phi(s))$ and $|\phi(0)|^{p} \geqslant \rho ;$

(3) $V\left(t_{k}, \phi(0)+I\left(t_{k}, \phi(0)\right)\right) \leqslant \lambda V\left(t_{k}^{-}, \phi(0)\right)$ for all $|\phi(0)|^{p} \geqslant$ $\rho$;

(4) there exists a positive constant $\rho_{1} \geqslant \rho$ such that if $|\phi(0)|^{p} \leqslant \rho$, then $\left|\phi(0)+I\left(t_{k}, \phi(0)\right)\right|^{p} \leqslant \rho_{1} ;$

(5) $\alpha=\sup _{k \in \mathbb{Z}}\left\{t_{k}-t_{k-1}\right\}<\infty, \alpha \gamma<1-\lambda$.

Then the system (1) is p-moment uniformly ultimately bounded.

Proof. We separate the proof into two parts. First, we show the $p$-moment uniform boundedness and then we give the ultimate uniform boundedness.

Step 1. Let $B_{1}>0$. Without loss of generality, we assume $B_{1} \geqslant$ $\rho_{1} \geqslant \rho$. Choose $B_{2}=B_{2}\left(B_{1}\right)$ such that $b B_{1}<\lambda a B_{2}$; then we can see $B_{2}>B_{1}$. 
Let $E\|\varphi\|_{\tau}^{p}<B_{1}$ and $t_{0} \in\left[t_{l-1}, t_{l}\right)$ for some positive integer l. Suppose $x(t)=x\left(t, t_{0}, \varphi\right)$ is a solution of system (1) with initial value $\varphi$ and its maximal interval of existence is $\left[t_{0}-\right.$ $\left.\tau, t_{0}+\beta\right)$ for some positive constant $\beta$. We will show that, for any $t \in\left[t_{0}-\tau, t_{0}+\beta\right), E|x(t)|^{p} \leqslant B_{2}$. By the way, if this statement is true, we know that the solution of system (1) is not explored in $\left[t_{0}, t_{0}+\beta\right)$, and the global existence of the solution follows.

For the sake of contradiction, suppose $E|x(t)|^{p} \geqslant B_{2}$ for some $t \in\left[t_{0}, t_{0}+\beta\right)$. Then there exists $\widehat{t}=\inf \left\{t \in\left[t_{0}-\tau, t_{0}+\right.\right.$ $\left.\beta)\left.|E| x(t)\right|^{p}>B_{2}\right\}$. Note that $E|x(t)|^{p} \leqslant E\|\varphi\|_{\tau}^{p} \leqslant B_{1}<B_{2}$ for $t \in\left[t_{0}-\tau, t_{0}\right]$; we see that $\widehat{t} \in\left(t_{0}, t_{0}+\beta\right)$ and $E|x(t)|^{p} \leqslant B_{2}$ for $t \in\left[t_{0}-\tau, \widehat{t}\right)$ and $E|x(\hat{t})|^{p} \geqslant B_{2}$.

Write $V(t, x(t))=V(t)$. For $t \in\left[t_{0}-\tau, t_{0}\right]$, we have $E V(t)$ $\leqslant b E|x(t)|^{p} \leqslant b E\|\varphi\|_{\tau}^{p} \leqslant b B_{1}<\lambda a B_{2}<a B_{2}$, and $E V(t) \geqslant$ $a E|x(\hat{t})|^{p} \geqslant a B_{2}$. Define $t^{*}=\inf \left\{t \in\left[t_{0}, \widehat{t}\right] \mid E V(t) \geqslant a B_{2}\right\}$ and then $t^{*} \in\left(t_{0}, \widehat{t}\right]$ and $E V(t)<a B_{2}$ for $t \in\left[t_{0}-\tau, t^{*}\right)$ and $E V\left(t^{*}\right) \geqslant a B_{2}$. $a B_{2}$.

We claim that $t^{*} \neq t_{k}$ for any $k \in \mathbb{N}$ and then $E V\left(t^{*}\right)=$

If it is not true, suppose $t^{*}=t_{k}$ for some $k$. If $E\left|x\left(t_{k}^{-}\right)\right|^{p} \geqslant$ $\rho$, then $a B_{2} \leqslant E V\left(t_{k}\right) \leqslant \lambda E V\left(t_{k}^{-}\right)<\lambda a B_{2}<a B_{2}$, which is a contradiction. If $E\left|x\left(t_{k}^{-}\right)\right|^{p}<\rho$, then $E\left|x\left(t_{k}\right)\right|^{p}=E \mid x\left(t_{k}^{-}\right)+$ $\left.I\left(t_{k}, x\left(t_{k}^{-}\right)\right)\right|^{p}<\rho_{1}<B_{1}$. Then $a B_{2}<E V\left(t_{k}\right)<b B_{1}<\lambda a B_{2}<$ $a B_{2}$, which is a contradiction.

Now we will proceed under two cases.

Case 1. Consider $t_{l-1} \leqslant t_{0}<t^{*}<t_{l}$.

Let $\bar{t}=\sup \left\{t \in\left[t_{0}, t^{*}\right] \mid E V(t) \leqslant \lambda a B_{2}\right\}$. Since $E V\left(t_{0}\right)<$ $b B_{1}<\lambda a B_{2}, E V\left(t^{*}\right)=a B_{2}>\lambda a B_{2}$, and $E V(t)$ is continuous on $\left[t_{0}, t^{*}\right]$, then $\bar{t} \in\left(t_{0}, t^{*}\right)$ and $E V(\bar{t})=\lambda a B_{2}$ and, when $t \in\left[\bar{t}, t^{*}\right], E V(t) \geqslant \lambda a B_{2}$. Hence, for $t \in\left[\bar{t}, t^{*}\right]$ and $s \in[-\tau, 0]$, we have

$$
\begin{gathered}
\lambda E V(t+s) \leqslant \lambda a B_{2} \leqslant E V(t), \\
b B_{1} \leqslant \lambda a B_{2} \leqslant E V(t) \leqslant b E|x(t)|^{p},
\end{gathered}
$$

and we can get

$$
E|x(t)|^{p} \geqslant B_{1} \geqslant \rho .
$$

Then, by virtue of condition (2), for $t \in\left[\bar{t}, t^{*}\right]$,

$$
\begin{gathered}
E \mathscr{L} V(t) \leqslant \gamma E V(t) \\
E V\left(t^{*}\right)-E V(\bar{t})=\int_{\bar{t}}^{t^{*}} E \mathscr{L} V(s) d s \\
\leqslant \int_{\bar{t}}^{t^{*}} \gamma E V(s) d s<\gamma \alpha a B_{2} .
\end{gathered}
$$

However,

$$
E V\left(t^{*}\right)-E V(\bar{t})=a B_{2}-\lambda a B_{2}=(1-\lambda) a B_{2}>\gamma \alpha a B_{2},
$$

which is contradiction. Then we get, in this case,

$$
E|x(t)|^{p} \leqslant B_{2} .
$$

Case 2. Consider $t_{k}<t^{*}<t_{k+1}$ for some $k \geqslant l$.

Note that $E V\left(t_{k}\right) \leqslant \lambda a B_{2}$. This inequality can be obtained by the following reason: if $E\left|x\left(t_{k}^{-}\right)\right|^{p} \geqslant \rho$, then $E V\left(t_{k}\right) \leqslant$ $\lambda E V\left(t_{k}^{-}\right) \leqslant \lambda a B_{2}$. If $E\left|x\left(t_{k}^{-}\right)\right|^{p}<\rho$, we get $E\left|x\left(t_{k}\right)\right|^{p}<\rho_{1}<B_{1}$, and then

$$
E V\left(t_{k}\right)<b B_{1}<\lambda a B_{2} .
$$

Define $\bar{t}=\sup \left\{t \in\left[t_{k}, t^{*}\right] \mid E V(t) \leqslant \lambda a B_{2}\right\}$, and then $\bar{t} \in\left[t_{k}, t^{*}\right), E V(\bar{t})=\lambda a B_{2}$, and $E V(t) \geqslant \lambda a B_{2}$ for $t \in\left[\bar{t}, t^{*}\right]$. The same argument as the one in Case 1 yields a contradiction. Therefore, in this case, we have, for any $t \in\left[t_{0}-\tau, \infty\right)$,

$$
E|x(t)|^{p} \leqslant B_{2} .
$$

Now we get that, under conditions (1) to condition (5), the solutions of (1) are $p$-moment uniformly bounded. That is, if $E\|\varphi\|_{\tau}^{p} \leqslant \rho_{1}$, there exists a constant $B>0$, such that $E\left|x\left(t, t_{0}, \varphi\right)\right|^{p} \leqslant B$ for all $t \geqslant t_{0}-\tau$, and, from the proof, we have $b \rho_{1}<\lambda a B$.

Step 2. Now, let $B_{3}>0$ and assume, without loss of generality, that $B_{3}>B$. Then, from the proof of uniform boundedness, there exists some $B_{2}=B_{2}\left(B_{3}\right)>B_{3}$ for which if $E\|\varphi\|_{\tau}^{p} \leqslant B_{3}$, then $E|x(t)|^{p} \leqslant B_{2}$ for $t \geqslant t_{0}-\tau$.

Take a constant $d$ satisfying $0<d \leqslant(1-\lambda-\gamma \tau) a B /(1-\gamma \tau)$; it is easy to verify that $0<d<(1-\lambda) a B$. Let $N=N\left(B_{3}\right)$ be the smallest positive integer for which $b B_{2}<a B+N d$ and $T=T\left(B_{3}\right)=\alpha+(\tau+\alpha)(N-1)$. Given a solution $x(t)=$ $x\left(t, t_{0}, \varphi\right)$ where $E\|\varphi\|_{\tau}^{p} \leqslant B_{3}$ and $t_{0} \in\left[t_{l-1}, t_{l}\right)$, we will show $E|x(t)|^{p} \leqslant B$ for $t \geqslant t_{0}+T$.

Given a constant $A$ satisfying $a B \leqslant A-d \leqslant b B_{2}$ and $j>l$, we will show that if $E V(t) \leqslant A$ for $t \in\left[t_{j}-\tau, t_{j}\right)$, then $E V(t) \leqslant A-d$ for $t \geqslant t_{j}$.

For the sake of contradiction, suppose that there exists some $t \geqslant t_{j}$ for which $E V(t)>A-d$ and define

$$
t^{*}=\inf \left\{t \geqslant t_{j} \mid E V(t)>A-d\right\},
$$

and we suppose $t^{*} \in\left[t_{k}, t_{k+1}\right)$ for some $k \in \mathbb{N}$. We can get $E V(t) \leqslant A-d$ for $t \in\left[t_{j}-\tau, t^{*}\right)$ and $E V\left(t^{*}\right) \geqslant A-d$.

We claim that $E V\left(t_{k}\right) \leqslant \lambda A$. The fact follows that if $E\left|x\left(t_{k}^{-}\right)\right|^{p} \geqslant \rho$, then $E V\left(t_{k}\right) \leqslant \lambda E V\left(t_{k}^{-}\right) \leqslant \lambda A$. If $E\left|x\left(t_{k}^{-}\right)\right|^{p}<\rho$ and we have $E\left|x\left(t_{k}\right)\right|^{p} \leqslant \rho_{1}$, then $E V\left(t_{k}\right) \leqslant b \rho<b B \leqslant \lambda a B \leqslant$ $\lambda A$.

Now, since $a B \leqslant A$, we have $\lambda A=A-(1-\lambda) A<A-(1-$ d) $a B<A-d$ and $E V\left(t_{k}\right)<A-d$. This implies that $t^{*} \neq t_{k}$; that is, $t^{*} \in\left(t_{k}, t_{k+1}\right)$ and $E V\left(t^{*}\right)=A-d$ since $E V(t)$ is continuous at $t^{*}$. Also, for $t \in\left[t_{k}, t^{*}\right]$, we have $E V(t) \leqslant A-d$. Define

$$
\bar{t}=\sup \left\{t \in\left[t_{k}, t^{*}\right] \mid E V(t) \leqslant \lambda(A-d)\right\} .
$$

Since $E V\left(t^{*}\right)=A-d>\lambda A>\lambda(A-d)$, we have $\bar{t} \in\left[t_{k}, t^{*}\right)$ and $E V(\bar{t})=\lambda(A-d)$ and $E V(t) \geqslant \lambda(A-d)$ for $t \in\left[\bar{t}, t^{*}\right]$. Then, if $t \in\left[\bar{t}, t^{*}\right]$ and $s \in[-\tau, 0]$,

$$
\begin{gathered}
\lambda E V(t+s) \leqslant \lambda(A-d)<E V(t), \\
b E|x(t)|^{p}>E V(t)>\lambda(A-d)>\lambda a B>b \rho,
\end{gathered}
$$


which yields $E|x(t)|^{p}>\rho$. Then, in light of condition (2),

$$
E \mathscr{L} V(t) \leqslant \gamma E V(t) \text {. }
$$

In terms of Itô formula,

$$
\begin{aligned}
E V\left(t^{*}\right)-E V(\bar{t}) & =\int_{\bar{t}}^{t^{*}} E \mathscr{L} V(s) d s \\
& \leqslant \int_{\bar{t}}^{t^{*}} \gamma E V(s) d s \leqslant \gamma \alpha(A-d) .
\end{aligned}
$$

But

$$
E V\left(t^{*}\right)-E V(\bar{t})=A-d-\lambda(A-d)>\gamma \alpha(A-d),
$$

and this contradiction proves that $E V(t)<A-d$ for all $t \geqslant$ $t_{j}$.

Now we define a sequence $t_{k^{(i)}} \in\left\{t_{k}, k=l, l+1, \ldots\right\}$, satisfying $t_{k^{(1)}}=t_{l}$ and $t_{k^{(i)}-1}-\tau \leqslant t_{k^{(i-1)}} \leqslant t_{k^{(i)}}-\tau$, and then we have $t_{k^{(i)}} \leqslant t_{k^{(i)}-1}+\alpha \leqslant t_{k^{(i-1)}}+\tau+\alpha$. By induction, we get $t_{k^{(N)}} \leqslant t_{0}+\alpha+(\tau+\alpha)(N-1)=t_{0}+T$. We know that when $t \in\left[t_{0}-\tau, t_{l}\right)$, that is, $t \in\left[t_{0}-\tau, t_{k^{(1)}}\right), E V(t) \leqslant b B_{2}$; then by induction we get $E V(t) \leqslant b B_{2}-N d$ for $t \in\left[t_{k^{(N)}}, \infty\right)$ and then $E V(t) \leqslant a B$ for $t \in\left[t_{0}+T, \infty\right)$. Using condition (1), we get that $a E|x(t)|^{p} \leqslant E V(t) \leqslant a B$; that is,

$$
E|x(t)|^{p} \leqslant B
$$

Remark 3. Condition (2) means the system without impulse may be unbounded. If the impulsive effects satisfy condition (3) to condition (5), then this system can be bounded.

\section{Boundedness with Impulsive Disturbance}

In this section, we consider the case that the SDDS is bounded, and when the impulsive disturbance appears in the SDDS, then what restrictions should be added to the disturbance to maintain the boundedness. The result is stated as follows.

Theorem 4. Assume that there exist a positive function $V(t, x)$ and positive constants $a, b, c, p, \lambda_{1}, \lambda_{2}, \gamma$, where $1 \leqslant \lambda_{1}<\lambda_{2}$, such that

(1) $a|x|^{p} \leqslant V(t, x) \leqslant b|x|^{p}$ for any $(t, x)$;

(2) for $t \neq t_{k}$, any $s \in[-\tau, 0]$, and $\phi(s) \in P C\left([-\tau, 0], \mathbb{R}^{n}\right)$, $\mathscr{L} V(t, \phi(0)) \leqslant-\gamma V(t, \phi(0))$ whenever $\lambda_{2} V(t, \phi(0)) \geqslant$ $V(t+s, \phi(s))$ and $|\phi(0)|^{p} \geqslant \rho ;$

(3) $V\left(t_{k}, \phi(0)+I\left(t_{k}, \phi(0)\right)\right) \leqslant \lambda_{1} V\left(t_{k}^{-}, \phi(0)\right)$ for all $|\phi(0)|^{p} \geqslant \rho ;$

(4) there exists a positive constant $\rho_{1} \geqslant \rho$ such that if $|\phi(0)|^{p} \leqslant \rho$, then $\left|\phi(0)+I\left(\tau_{k}, \phi(0)\right)\right|^{p} \leqslant \rho_{1} ;$

(5) there exist positive constants $\mu$ and $\alpha$, such that $\mu \leqslant$ $t_{k}-t_{k-1} \leqslant \alpha$ and $\mu \gamma>\lambda_{2}-1$.

Then, the system (1) is p-moment uniformly ultimately bounded.
Proof. Step 1. Let $B_{1}>0$; without loss of generality, we assume $B_{1} \geqslant \rho_{1}$. Choose $B_{2}=B_{2}\left(B_{1}\right)$, such that $\lambda_{2} b B_{1}<a B_{2}$, and then we get $B_{2}>B_{1}$. Let $E\|\varphi\|_{\tau}^{p} \leqslant B_{1}$ and assume $t_{0} \in\left[t_{l-1}, t_{l}\right)$; moreover, we assume that (1) has a maximal interval of existence, $\left[t_{0}-\tau, t_{0}+\beta\right)$.

We will prove that $E|x(t)|^{p} \leqslant B_{2}$ for $t \in\left[t_{0}, t_{0}+\beta\right)$. This will show that $\beta=\infty$ and that solutions of (1) are uniformly bounded.

For the sake of contradiction, we suppose that $E|x(t)|^{p}>$ $B_{2}$ for some $t \in\left[t_{0}, t_{0}+\beta\right)$. Let $\widehat{t}=\inf \left\{t \in\left[t_{0}, t_{0}+\beta\right) \mid\right.$ $\left.E|x(t)|^{p}>B_{2}\right\}$. Note that $E|x(t)|^{p} \leqslant E\|\varphi\|_{\tau}^{p}<B_{1}<B_{2}$ for $t \in\left[t_{0}-\tau, t_{0}\right]$, and we get $\widehat{t} \in\left(t_{0}, t_{0}+\beta\right), E|x(t)|^{p} \leqslant B_{2}$ for $t \in\left[t_{0}-\tau, \widehat{t}\right)$ and $E|x(\hat{t})|^{p} \geqslant B_{2}$.

For $t \in\left[t_{0}-\tau, t_{0}\right]$, we have $E V(t) \leqslant b E|x(t)|^{p} \leqslant b E\|\varphi\|_{\tau}^{p} \leqslant$ $b B_{1}$ and then $E V(t) \leqslant \lambda_{2} E V(t) \leqslant \lambda_{2} b B_{1}<a B_{2}$. Particularly, $E V\left(t_{0}\right) \leqslant \lambda_{2} E V\left(t_{0}\right)<a B_{2}$ and $E V(\hat{t}) \geqslant a E|x(\hat{t})|^{p} \geqslant a B_{2}$.

Define $t^{*}=\inf \left\{t \in\left[t_{0}, \widehat{t}\right] \mid E V(t) \geqslant a B_{2}\right\}$ and then $t^{*} \in$ $\left(t_{0}, \widehat{t}\right], E V\left(t^{*}\right) \geqslant a B_{2}$, and $E V(t)<a B_{2}$ for $t \in\left[t_{0}-\tau, t^{*}\right)$.

Now we will proceed under two cases.

Case 1. Consider $t_{l-1} \leqslant t_{0}<t^{*}<t_{l}$.

Under this case, we have $E V\left(t^{*}\right)=a B_{2}$ because of the continuity of $V(t)$ on $\left(t_{k}, t_{k+1}\right)$ and $\lambda_{2} E V\left(t^{*}\right)=\lambda_{2} a B_{2}>a B_{2}$. Define $\bar{t}=\sup \left\{t \in\left[t_{0}, t^{*}\right] \mid \lambda_{2} E V(t) \leqslant a B_{2}\right\}$ and then $\bar{t} \neq t^{*}$, $\lambda_{2} E V(\bar{t})=a B_{2}$, and $\lambda_{2} E V(t) \geqslant a B_{2}$ for $t \in\left[\bar{t}, t^{*}\right]$. Therefor, for any $t \in\left[\bar{t}, t^{*}\right]$ and $s \in[-\tau, 0]$, we have $E V(t+s) \leqslant a B_{2}<$ $\lambda_{2} E V(t)$ and $\lambda_{2} b B_{1}<a B_{2}<\lambda_{2} E V(t)$, which yields $E V(t)>$ $b B_{1}$, and then we have $E|x(t)|^{p}>B_{1} \geqslant \rho$. Using condition (2), we have, when $t \in\left[\bar{t}, t^{*}\right]$,

$$
E \mathscr{L} V(t) \leqslant-\gamma E V(t)
$$

By virtue of Itô formula, we have

$$
E V\left(t^{*}\right)-E V(\bar{t})=\int_{\bar{t}}^{t^{*}} E \mathscr{L V}(s) d s \leqslant \int_{\bar{t}}^{t^{*}}-\gamma E V(s) d s \leqslant 0 .
$$

However,

$$
E V\left(t^{*}\right)=a B_{2}>\frac{a B_{2}}{\lambda_{2}}=E V(\bar{t})
$$

This contradiction gives

$$
E|x(t)|^{p} \leqslant B_{2} \quad \text { for } t \in\left[t_{0}, t_{0}+\beta\right) .
$$

Case 2. Consider $t_{k} \leqslant t^{*}<t_{k+1}$ for some $k \geqslant l$.

We first show $\lambda_{2} E V\left(t_{k}^{-}\right) \leqslant a B_{2}$. We have two situations to contemplate: $k=l$ and $k>l$.

If $k=l$, we suppose $\lambda_{2} E V\left(t_{l}^{-}\right)>a B_{2}$. Define $\bar{t}=\sup \{t \epsilon$ $\left.\left[t_{0}, t_{l}\right) \mid \lambda_{2} E V(t) \leqslant a B_{2}\right\}$ and then $\bar{t} \in\left(t_{0}, t_{l}\right)$ and $\lambda_{2} E V(\bar{t})=$ $a B_{2}$. In light of the definition of $\bar{t}$, we have, for $t \in\left[\bar{t}, t_{l}\right)$ and $s \in[-\tau, 0]$,

$$
\lambda_{2} E V(t) \geqslant a B_{2} \geqslant E V(t+s)
$$

and, for $t \in\left[\bar{t}, t_{l}\right)$,

$$
E|x(t)|^{p} \geqslant B_{1} \geqslant \rho .
$$


By virtue of condition (2), an analogous calculation of $E V\left(t_{l}^{-}\right)-E V(\bar{t})$ yields $E V\left(t_{l}^{-}\right) \leqslant E V(\bar{t})$; then we get

$$
a B_{2}<\lambda_{2} E V\left(t_{l}^{-}\right) \leqslant \lambda_{2} E V(\bar{t})=a B_{2} .
$$

If $k>l$, we suppose $\lambda_{2} E V\left(t_{k}^{-}\right)>a B_{2}$. We will proceed under two subcases.

Subcase 1. Consider $\lambda_{2} E V(t)>a B_{2}$ for all $t \in\left[t_{k-1}, t_{k}\right)$.

Under this situation, we have $\lambda_{2} E V(t)>a B_{2} \geqslant E V(t+s)$ and $E|x(t)|^{p} \geqslant \rho$ for all $t \in\left[t_{k-1}, t_{k}\right)$ and $s \in[-\tau, 0]$. In terms of condition (2), an analogous discussion as done in Case 1 gives

$$
\begin{aligned}
E V\left(t_{k}^{-}\right)-E V\left(t_{k-1}\right) & =\int_{t k-1}^{t_{k}^{-}} E \mathscr{L V}(s) d s \\
& \leqslant \int_{t k-1}^{t_{k}^{-}}-\gamma E V(s) d s \leqslant-\gamma \mu \frac{a B_{2}}{\lambda_{2}} .
\end{aligned}
$$

However, by virtue of condition (5),

$$
\begin{aligned}
E V\left(t_{k}^{-}\right)-E V\left(t_{k-1}\right) & \geqslant \frac{a B_{2}}{\lambda_{2}}-a B_{2}=\left(\frac{1}{\lambda_{2}}-1\right) a B_{2} \\
& >-\gamma \mu \frac{a B_{2}}{\lambda_{2}} .
\end{aligned}
$$

This contradiction implies

$$
\lambda_{2} E V\left(t_{k}^{-}\right) \leqslant a B_{2} \text { for } t_{k} \leqslant t^{*}<t_{k+1}, k \geqslant l .
$$

Subcase 2. Consider $\lambda_{2} E V(t) \leqslant a B_{2}$ for some $t \in\left[t_{k-1}, t_{k}\right)$.

Define $\bar{t}=\sup \left\{t \in\left[t_{k-1}, t_{k}\right) \mid \lambda_{2} E V(t) \leqslant a B_{2}\right\}$ and then $\bar{t} \in\left[t_{k-1}, t_{k}\right)$ and $\lambda_{2} E V(\bar{t})=a B_{2}$. Using the definition of $\bar{t}$, we get, for $t \in\left[\bar{t}, t_{k}\right)$ and $s \in[-\tau, 0], \lambda_{2} E V(t) \geqslant a B_{2} \geqslant E V(t+s)$. Since $\lambda_{2} E V(t) \geqslant a B_{2}$, using the fact $\rho_{1} \geqslant \rho, \lambda_{2} b B_{1}<a B_{2}$ and $b|x|^{p} \geqslant V(t, x)$, we can get $E|x(t)|^{p} \geqslant \rho$. By virtue of condition (2), we get, for $t \in\left[\bar{t}, t_{k}\right)$,

$$
E \mathscr{L} V(t) \leqslant-\gamma E V(t)
$$

An analogous discussion as done in the case $k=l$ gives $E V(\bar{t}) \geqslant E V\left(t_{k}^{-}\right)$. Then we have

$$
a B_{2}<\lambda_{2} E V\left(t_{k}^{-}\right) \leqslant \lambda_{2} E V(\bar{t})=a B_{2} .
$$

This contradiction gives

$$
\lambda_{2} E V\left(t_{k}^{-}\right) \leqslant a B_{2} \text { for } t_{k} \leqslant t^{*}<t_{k+1}, k \geqslant l .
$$

Now we claim $E V\left(t_{k}\right)<a B_{2}$. If $E\left|x\left(t_{k}^{-}\right)\right|^{p} \geqslant \rho$, we get $E V\left(t_{k}\right) \leqslant \lambda_{1} E V\left(t_{k}^{-}\right)<\lambda_{2} E V\left(t_{k}^{-}\right)<a B_{2}$. If $E\left|x\left(t_{k}^{-}\right)\right|^{p}<\rho$, we get $E V\left(t_{k}\right) \leqslant b \rho_{1}<b B_{1}<\lambda_{2} b B_{1}<a B_{2}$. That is, the following inequality holds:

$$
E V\left(t_{k}\right)<a B_{2}
$$

Since $E V\left(t^{*}\right) \geqslant a B_{2}$, we have $t^{*} \neq t_{k}$ and $E V\left(t^{*}\right)=a B_{2}$. If $\lambda_{2} E V\left(t^{*}\right) \geqslant a B_{2}$ for all $t \in\left[t_{k}, t^{*}\right]$, then let $\bar{t}=t_{k}$ and we have $E V(\bar{t})<a B_{2}$. Otherwise, let $\bar{t}=\sup \left\{t \in\left[t_{k}, t^{*}\right) \mid\right.$ $\left.\lambda_{2} E V(t) \leqslant a B_{2}\right\}$, and we have $E V(\bar{t})<\lambda_{2} E V(\bar{t})=a B_{2}$. Since $E V\left(t^{*}\right)=a B_{2}$, we get $\bar{t} \in\left[t_{k}, t^{*}\right)$. Moreover, for $t \in\left[\bar{t}, t^{*}\right]$, we have $\lambda_{2} E V(t) \geqslant a B_{2}>E V(t+s)$ and, by virtue of $\lambda_{2} b B_{1}<$ $a B_{2}<\lambda_{2} E V(t)$, we obtain $E V(t)>b B_{1}$ and then $E|x(t)|^{p}>$ $B_{1}>\rho$. In terms of condition (2) and Itô formula, we can obtain $E V(\bar{t}) \geqslant E V\left(t^{*}\right)$. But $E V(\bar{t})<a B_{2}=E V\left(t^{*}\right)$, which is a contradiction and yields

$$
E|x(t)|^{p} \leqslant B_{2} \quad \text { for } t \in\left[t_{0}, t_{0}+\beta\right) .
$$

Now we get that, under condition (1) to condition (5), the solutions of (1) are $p$-moment uniformly bounded. Then we know that if $E\|\varphi\|_{\tau}^{p} \leqslant \rho_{1}$, there exists a constant $B>0$, such that $E\left|x\left(t, t_{0}, \varphi\right)\right|^{p} \leqslant B$ for all $t \geqslant t_{0}-\tau$, and, from the above proof, we have $\lambda_{2} b \rho_{1}<a B$.

Step 2. Now, let $B_{3}>0$ and assume, without loss of generality, that $B_{3}>B$. Then, from the proof of uniform boundedness, there exists a constant $B_{2}=B_{2}\left(B_{3}\right)>B_{3}$ for which if $E\|\varphi\|_{\tau}^{p} \leqslant$ $B_{3}$, then $E|x(t)|^{p} \leqslant B_{2}$ for $t \geqslant t_{0}-\tau$.

Take a constant $d$ satisfying $0<d \leqslant \min \left\{a B-b \rho_{1},\left(\left(\lambda_{2}-\right.\right.\right.$ $\left.\left.\left.\lambda_{1}\right) / \lambda_{2}\right) a B\right\}, N=\min \left\{n>\left(\left(b B_{2}-a B\right) / d\right)\right\}$, and $T=\alpha+(2 N-$ 1) $(\alpha+\tau)$.

Let $x(t)=x\left(t, t_{0}, \varphi\right)$ be a solution of (1) with $E\|\varphi\|_{\tau}^{p} \leqslant B_{3}$, $t_{0} \in\left[t_{l-1}, t_{l}\right)$. We will show $E|x(t)|^{p} \leqslant B$ for $t \geqslant t_{0}+T$.

Given a positive number $A$ satisfying $a B \leqslant A \leqslant b B_{2}$ and $j \geqslant l$, we will show that if $E V(t) \leqslant A$ for $t \in\left[t_{j}-\tau, t_{j}\right)$ and $\lambda_{2} E V\left(t_{j}^{-}\right) \leqslant A$, then $E V(t) \leqslant A$ for $t \geqslant t_{j}$ and $\lambda_{2} E V\left(t_{j+1}^{-}\right) \leqslant$ A.

For the sake of contradiction, suppose that there exists a constant $t \in\left[t_{j}, t_{j+1}\right)$ for which $E V(t)>A$ and define

$$
t^{*}=\inf \left\{t \in\left[t_{j}, t_{j+1}\right) \mid E V(t) \geqslant A\right\} .
$$

Note that $E V\left(t_{j}\right)<A$, and we have that if $E\left|x\left(t_{j}^{-}\right)\right|^{p} \geqslant \rho$, then $E V\left(t_{k}\right) \leqslant \lambda_{1} E V\left(t_{j}^{-}\right)<\lambda_{2} E V\left(t_{j}^{-}\right) \leqslant A$. If $E\left|x\left(t_{k}^{-}\right)\right|^{p}<\rho$, we have $E V\left(t_{j}\right) \leqslant b \rho_{1}<\lambda_{2} b \rho_{1}<a B \leqslant A$. Then we get $t^{*} \neq t_{j}$, $E V\left(t^{*}\right)=A$, and $E V(t) \leqslant A$ for $t \in\left(t_{j}, t_{j+1}\right]$.

If $\lambda_{2} E V(t)>A$ for all $t \in\left[t_{j}, t_{j+1}\right)$, we let $\bar{t}=t_{j}$, and then $E V(\bar{t})=E V\left(t_{j}\right)<A$. Otherwise, let $\bar{t}=\sup \left\{t \in\left[t_{j}, t^{*}\right]\right.$ | $\left.\lambda_{2} E V(t) \leqslant A\right\}$, and we get $E V(\bar{t}) \leqslant \lambda_{2} E V(\bar{t})=A$. Since $\lambda_{2} E V\left(t^{*}\right)=\lambda_{2} A>A, \bar{t} \neq t^{*}$. For $t \in\left[\bar{t}, t^{*}\right]$ and $s \in[-\tau, 0]$, we have $\lambda_{2} E V(t) \geqslant A \geqslant E V(t+s)$. Moreover, for $t \in\left[\bar{t}, t^{*}\right]$,

$$
\lambda_{2} E V(t) \geqslant A \geqslant a B>\lambda_{2} b \rho_{1}
$$

and we get $E|x(t)|^{p} \geqslant \rho_{1} \geqslant \rho$. By virtue of condition (2) and Itô formula, we can get $E V(\bar{t}) \geqslant E V\left(t^{*}\right)$. However, $E V\left(t^{*}\right)=$ $A>E V(\bar{t})$.

Now we have proven $E V(t) \leqslant A$ for $t \in\left[t_{j}, t_{j+1}\right)$, and we are on the position to show $\lambda_{2} E V\left(t_{j+1}^{-}\right) \leqslant A$. This will follow in the same way as the arguments used in the proof of uniform boundedness, where we show $\lambda_{2} E V\left(t_{k}^{-}\right) \leqslant a B_{2}$ for the case $k>l$; we just need to replace $k$ by $j+1$ and $a b_{2}$ by $A$.

By induction, we get that if $E V(t) \leqslant A$ for $t \in\left[t_{j}-\tau, t_{j}\right)$ and $\lambda_{2} E V\left(t_{j}^{-}\right) \leqslant A$, then $E V(t) \leqslant A$ for all $t \geqslant t_{j}$ and $\lambda_{2} E V\left(t_{k}^{-}\right) \leqslant A$ for $k \geqslant j+1$. 
Next, we will show $E V(t) \leqslant A-d$ for $t \in\left[t_{j+1}, t_{j+2}\right)$, if $E V(t) \leqslant A$ for all $t \geqslant t_{j}$ and $\lambda_{2} E V\left(t_{k}^{-}\right) \leqslant A, k \geqslant j$.

We first show $E V\left(t_{j+1}\right) \leqslant A-d$. This can be easily verified under two situations: iIf $E\left|x\left(t_{j+1}^{-}\right)\right|^{p} \leqslant \rho$, we have $E V\left(t_{j+1}\right) \leqslant$ $b \rho_{1} \leqslant a B-d \leqslant A-d$; if $E\left|x\left(t_{j+1}^{-}\right)\right|^{p}>\rho, E V\left(t_{j+1}\right)<$ $\lambda_{1} E V\left(t_{j+1}\right)=\left(\lambda_{1} / \lambda_{2}\right) \lambda_{2} E V\left(t_{j+1}^{-}\right) \leqslant\left(\lambda_{1} / \lambda_{2}\right) A<A-d$.

In order to verify $E V(t) \leqslant A-d$ for all $t \in\left[t_{j+1}, t_{j+2}\right)$, suppose that $E V(t)>A-d$ for some $t \in\left[t_{j+1}, t_{j+2}\right)$. Let $t^{*}=\inf \left\{t \in\left[t_{j+1}, t_{j+2}\right) \mid E V(t) \geqslant A-d\right\}$; we know $t^{*} \neq t_{j+1}$ and then $E V\left(t^{*}\right)=A-d$ and $\lambda_{2} E V\left(t^{*}\right)=\lambda_{2}(A-d)>A$.

If $\lambda_{2} E V(t)>A$ for all $t \in\left[t_{j+1}, t^{*}\right]$, let $\bar{t}=t_{j+1}, E V(\bar{t})=$ $E V\left(t_{j+1}\right)<A-d$.

If $\lambda_{2} E V(t)>A$ for some $t \in\left(t_{j+1}, t^{*}\right]$, let $\bar{t}=\sup \{t \in$ $\left.\left[t_{j+1}, t^{*}\right] \mid \lambda_{2} E V(t) \leqslant A\right\}$ and we know $\bar{t} \neq t^{*}, E V(\bar{t})=A / \lambda_{2}$.

For $t \in\left[\bar{t}, t^{*}\right]$ and $s \in[-\tau, 0], \lambda_{2} E V(t) \geqslant A>A-d>$ $E V(t+s)$ and $E V(t) \geqslant A / \lambda_{2}>a B / \lambda_{2}>b \rho_{1}$, and we get $E|x(t)|^{p}>\rho_{1} \geqslant \rho$. In terms of condition (2) and Itô formula, we can get $E V\left(t^{*}\right)<E V(\bar{t})$. However, $E V\left(t^{*}\right)=A-d>$ $E V(\bar{t})$, which yields

$$
E V(t) \leqslant A-d .
$$

Applying our results to successive intervals of the form $\left[t_{k}, t_{k+1}\right)$ for $k \geqslant j+1$, we can get $E V(t) \leqslant A-d$ for $t \geqslant t_{j+1}$.

Now we need a fact $\lambda_{2} E V\left(t_{j+2}^{-}\right) \leqslant A-d$. This can be verified just as we did in the proof of uniform boundedness, where we show $\lambda_{2} E V\left(t_{k}^{-}\right) \leqslant a B_{2}$ for the case $k>l$.

Take $t_{k^{(i)}} \in\left\{t_{j}, j=l, l+1, \ldots\right\}$ satisfying $t_{k^{(i-1)}}+\tau \leqslant t_{k^{(i)}} \leqslant$

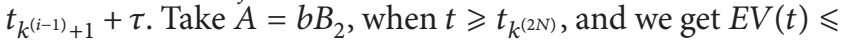
$b B_{2}-N d<a B$. Since $t_{k^{(2 N)}} \leqslant t_{k^{(1)}}+(2 N-1)(\alpha+\tau) \leqslant t_{0}+\alpha+$ $(2 N-1)(\alpha+\tau)=t_{0}+T$, we have $E V(t) \leqslant a B$ when $t>t_{0}+T$. By virtue of condition (1), $E|x(t)|^{p} \leqslant B$ for $t \geqslant t_{0}+T$, which completes the proof.

Remark 5. Theorem 4 considers that a bounded system without impulse can tolerate what kind of impulsive effects to hold the boundedness. It is not surprising that condition (3) to condition (5) should be satisfied: the interval of impulsive moments $(\mu)$ should be large and impulsive strength $\left(\lambda_{1}\right)$ should be small.

\section{Examples}

In this section, we present two examples to illustrate our results.

Example 1. Consider the following impulsive stochastic delay differential system:

$$
\begin{gathered}
d x(t)=\left(\frac{1}{2} x(t)+\frac{1}{2 x(t)}\right) d t+x\left(t-\frac{1}{20}\right) d B(t), \\
t>0, \quad t \neq \frac{k}{10}, \quad k=1,2, \ldots, \\
x\left(\frac{k}{10}\right)=\frac{\sqrt{2}}{2} x\left(\left(\frac{k}{10}\right)^{-}\right),
\end{gathered}
$$

where $B(t)$ is a one-dimension Brownian motion.

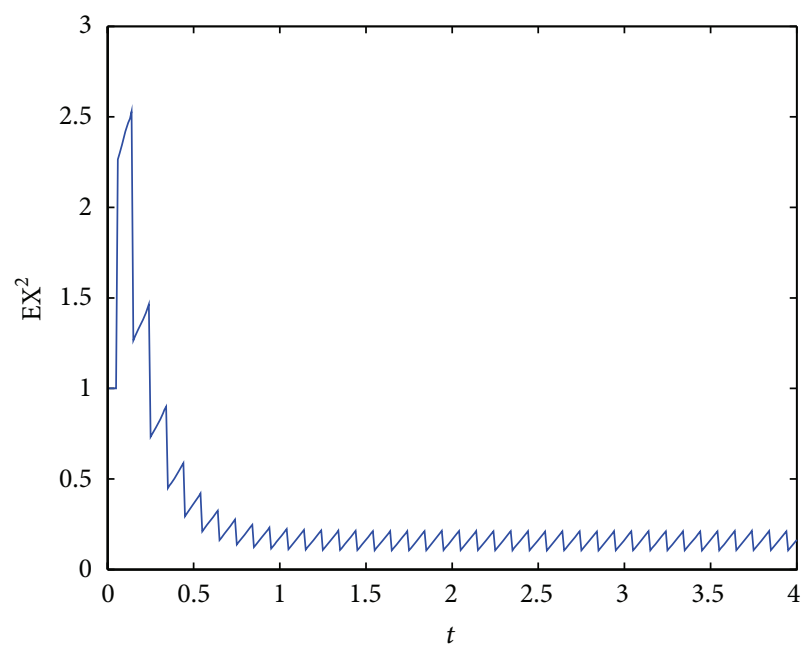

FIGURE 1: Mean square uniform ultimate boundedness of solution of system (36).

Define $V(t, x)=x^{2}$; the smoothness requirement is satisfied. Let $a=b=1$ and $p=2$; condition (1) of Theorem 2 follows. For any solution $x(t)$ of system (36), we have

$$
\begin{aligned}
\mathscr{L} V(t, x) & =2 x\left(\frac{1}{2} x(t)+\frac{1}{2 x(t)}\right)+x^{2}\left(t-\frac{1}{20}\right) \\
& =x^{2}(t)+1+x^{2}\left(t-\frac{1}{20}\right) .
\end{aligned}
$$

Take $\lambda=1 / 2$; condition ( 3 ) of Theorem 2 is satisfied.

Now let $\rho=1$; then, when $|x(t)|^{2} \geqslant 1$ and $V(t, x) \geqslant$ $\lambda V(t, x(t-\tau))$, that is, $x^{2}(t) \geqslant(1 / 2) x^{2}(t-1 / 20)$, we have

$$
\mathscr{L} V(t, x) \leqslant x^{2}(t)+x^{2}(t)+2 x^{2}(t)=4 x^{2}(t)=4 V(t, x) .
$$

Then let $\gamma=4$; condition (2) of Theorem 2 is verified. 1.

Condition (4) of Theorem 2 can be verified by taking $\rho_{1}=$

Take $\alpha=1 / 10$ and then $\alpha \gamma=(1 / 10) \times 4=2 / 5<1 / 2=$ $1-\lambda$; condition (5) of Theorem 2 is verified.

Therefore, according to Theorem 2, solutions of system (36) are mean square uniformly ultimately bounded. The boundedness can be read from Figure 1, where we take initial condition $x(t)=1, t \in[-1 / 20,0]$.

To see the contribution of impulsive effect on boundedness, we consider the following system:

$$
\begin{array}{r}
d x(t)=\left(\frac{1}{2} x(t)+\frac{1}{2 x(t)}\right) d t+x\left(t-\frac{1}{20}\right) d B(t), \\
t>0,
\end{array}
$$

which is the situation of system (36) without impulses. It is easy to be verified that system (39) is unbounded; see Figure 2, where we also take initial condition $x(t)=1, t \in$ $[-1 / 20,0]$.

Now we give another example to illustrate the correctness of Theorem 4. 


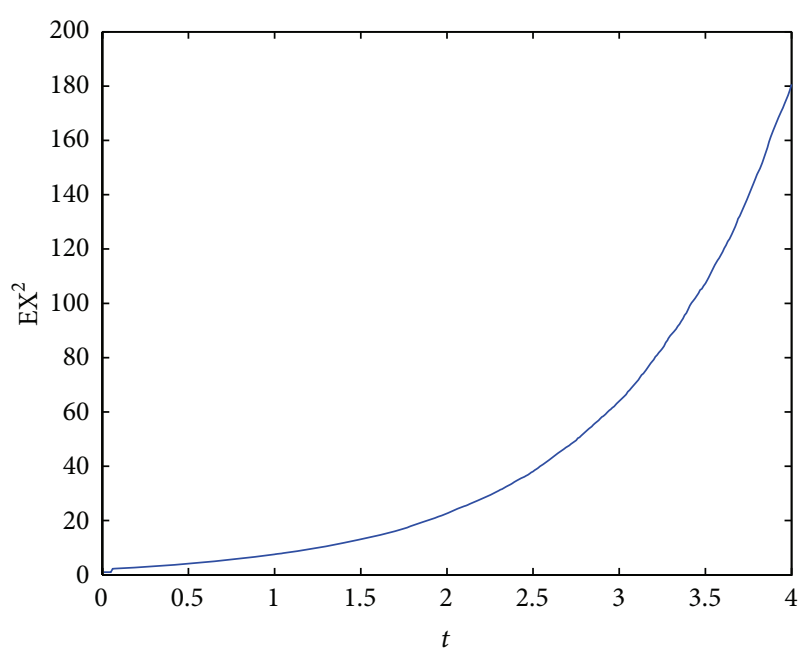

FIgURE 2: Unboundedness of solution of system (39).

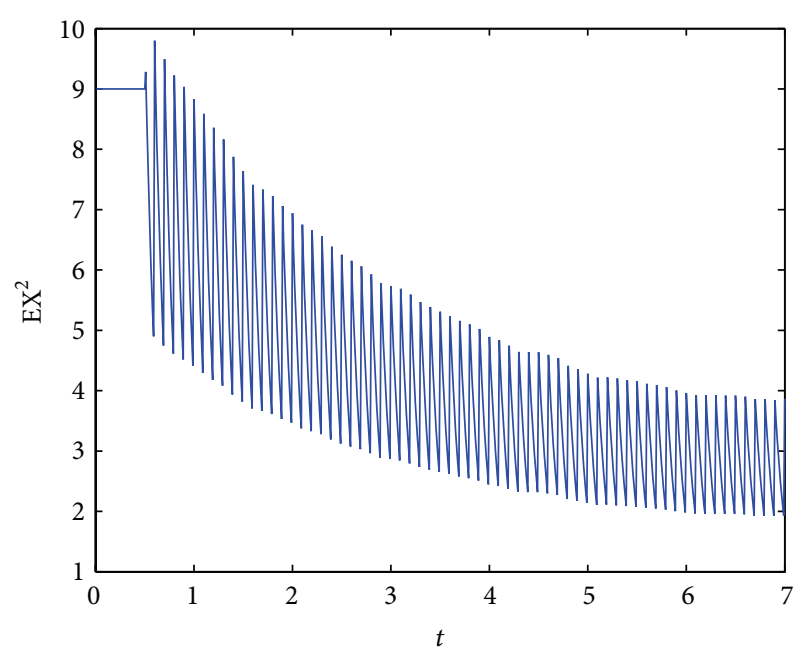

FIgURE 3: Mean square uniform ultimate boundedness of solution of system (40).

Example 2. Consider

$$
\begin{gathered}
d x(t)=\left(-4 x(t)+\frac{1}{2 x(t)}\right) d t+x\left(t-\frac{1}{2}\right) d B(t), \\
t>0, \quad t \neq 2 k, \quad k=1,2, \ldots, \\
x(2 k)=\sqrt{2} x\left((2 k)^{-}\right),
\end{gathered}
$$

where $B(t)$ is a one-dimension Brownian motion.

Define $V(t, x)=x^{2}$; the smoothness requirement is satisfied. Let $a=b=1$ and $p=2$; condition (1) of Theorem 4 follows. For any solution $x(t)$ of system (40), we have

$$
\begin{aligned}
\mathscr{L} V(t, x) & =2 x\left(-4 x(t)+\frac{1}{2 x(t)}\right)+x^{2}\left(t-\frac{1}{2}\right) \\
& =-8 x^{2}(t)+1+x^{2}\left(t-\frac{1}{20}\right) .
\end{aligned}
$$

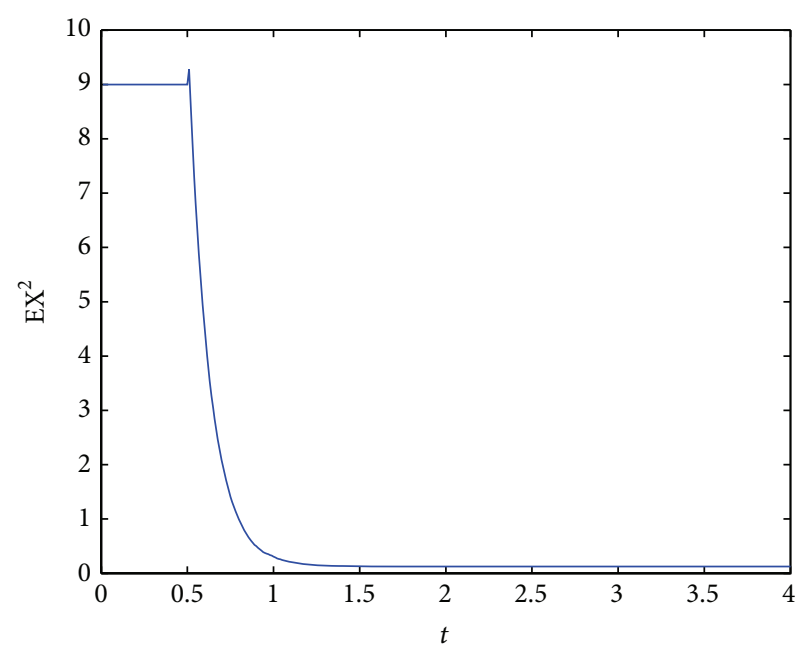

FIgURE 4: Simulation of system (43).

Take $\lambda_{1}=2$, condition (3) of Theorem 4 is satisfied.

Now let $\rho=1$ and $\lambda_{2}=3$; then, when $|x(t)|^{2} \geqslant 1$ and $V(t, x) \geqslant \lambda_{2} V(t, x(t-\tau))$, that is, $3 x^{2}(t) \geqslant x^{2}(t-1 / 2)$, we have

$$
\begin{aligned}
\mathscr{L} V(t, x) & \leqslant-8 x^{2}(t)+x^{2}(t)+3 x^{2}(t) \\
& =-4 x^{2}(t)=-4 V(t, x) .
\end{aligned}
$$

Then, let $\gamma=4$; condition (2) of Theorem 2 is verified.

Condition (4) of Theorem 2 can be verified by taking $\rho_{1}=2$.

Take $\mu=2$ and then $\mu \gamma=2 \times 8=16>3-1=\lambda_{2}-1$ and condition (5) of Theorem 4 is verified.

Therefore, according to Theorem 4, solutions of system (40) are mean square uniformly ultimately bounded. The boundedness can be seen in Figure 3, where we take initial condition $x(t)=3, t \in[-1 / 2,0]$.

We also present the simulation of system (40) without impulsive effects; that is,

$$
d x(t)=\left(-4 x(t)+\frac{1}{2 x(t)}\right) d t+x\left(t-\frac{1}{2}\right) d B(t), \quad t>0 .
$$

The property of system (43) can be read from Figure 4, where we take initial condition $x(t)=3, t \in[-1 / 2,0]$.

\section{Conflict of Interests}

The authors declare that there is no conflict of interests regarding to the publications of this paper.

\section{Acknowledgments}

This work is supported by the foundation under Grant HIT.IBRSEM.A.2014015 and by the National Natural Scientific Foundation of China under Grants 11271101, 61104193, and 61333001 . 


\section{References}

[1] L. Zu, D. Jiang, and D. O'Regan, "Stochastic permanence, stationary distribution and extinction of a single-species nonlinear diffusion system with random perturbation," Abstract and Applied Analysis, vol. 2014, Article ID 320460, 14 pages, 2014.

[2] E. Miao, H. Shu, and Y. Che, "Practical Stability in the pth Mean for Itô Stochastic Differential Equations," Mathematical Problems in Engineering, vol. 2010, Article ID 380304, 12 pages, 2012.

[3] X. Liu and Q. Wang, "The method of Lyapunov functionals and exponential stability of impulsive systems with time delay," Nonlinear Analysis: Theory, Methods \& Applications, vol. 66, no. 7, pp. 1465-1484, 2007.

[4] M. de la Sen, "Global stability of polytopic linear time-varying dynamic systems under time-varying point delays and impulsive controls," Mathematical Problems in Engineering, vol. 2010, Article ID 693958, 33 pages, 2010.

[5] X. Li, H. Akca, and X. Fu, "Uniform stability of impulsive infinite delay differential equations with applications to systems with integral impulsive conditions," Applied Mathematics and Computation, vol. 219, no. 14, pp. 7329-7337, 2013.

[6] Y. Zhang and J. Sun, "Boundedness of the solutions of impulsive differential systems with time-varying delay," Applied Mathematics and Computation, vol. 154, no. 1, pp. 279-288, 2004.

[7] L. Berezansky and E. Braverman, "Exponential boundedness of solutions for impulsive delay differential equations," Applied Mathematics Letters, vol. 9, no. 6, pp. 91-95, 1996.

[8] I. M. Stamova, "Lyapunov method for boundedness of solutions of nonlinear impulsive functional differential equations," Applied Mathematics and Computation, vol. 177, no. 2, pp. 714719, 2006.

[9] P. Cheng, F. Deng, and L. Wang, "The method of Lyapunov function and exponential stability of impulsive delay systems with delayed impulses," Mathematical Problems in Engineering, vol. 2013, Article ID 458047, 7 pages, 2013.

[10] X. Liu and G. Ballinger, "Boundedness for impulsive delay differential equations and applications to population growth models," Nonlinear Analysis: Theory, Methods \& Applications, vol. 53, no. 7-8, pp. 1041-1062, 2003.

[11] I. M. Stamova, "Boundedness of impulsive functional differential equations with variable impulsive perturbations," Bulletin of the Australian Mathematical Society, vol. 77, no. 2, pp. 331-345, 2008.

[12] I. Stamova, "Eventual stability and eventual boundedness for impulsive differential equations with "supremum"', Mathematical Modelling and Analysis, vol. 16, no. 2, pp. 304-314, 2011.

[13] A. Li and X. Song, "Stability and boundedness of nonlinear impulsive systems in terms of two measures via perturbing Lyapunov functions," Journal of Mathematical Analysis and Applications, vol. 375, no. 1, pp. 276-283, 2011.

[14] B. Chen and W. Zhang, "Stochastic $\mathrm{H}_{2} / \mathrm{H}_{\mathrm{\infty}}$ control with statedependent noise," IEEE Transactions on Automatic Control, vol. 49, no. 1, pp. 45-57, 2004.

[15] W. Zhang, H. Zhang, and B. Chen, "Generalized Lyapunov equation approach to state-dependent stochastic stabilization/detectability criterion," IEEE Transactions on Automatic Control, vol. 53, no. 7, pp. 1630-1642, 2008.

[16] W. Zhang and B. Chen, "H-representation and applications to generalized Lyapunov equations and linear stochastic systems," IEEE Transactions on Automatic Control, vol. 57, no. 12, pp. 3009-3022, 2012.
[17] F. Wu and P. E. Kloeden, "Mean-square random attractors of stochastic delay differential equations with random delay," Discrete and Continuous Dynamical Systems B, vol. 18, no. 6, pp. 1715-1734, 2013.

[18] B. Song, J. H. Park, Z. Wu, and Y. Zhang, "New results on delay-dependent stability analysis for neutral stochastic delay systems," Journal of the Franklin Institute: Engineering and Applied Mathematics, vol. 350, no. 4, pp. 840-852, 2013.

[19] X. Li, J. Zou, and E. Zhu, "pth moment exponential stability of impulsive stochastic neural networks with mixed delays," Mathematical Problems in Engineering, vol. 2012, Article ID 175934, 20 pages, 2012.

[20] K. Wu and X. Ding, "Stability and stabilization of impulsive stochastic delay differential equations," Mathematical Problems in Engineering, vol. 2012, Article ID 176375, 16 pages, 2012.

[21] X. Wu, W. Zhang, and Y. Tang, "pth moment stability of impulsive stochastic delay differential systems with Markovian switching," Communications in Nonlinear Science and Numerical Simulation, vol. 18, no. 7, pp. 1870-1879, 2013.

[22] P. Cheng, F. Deng, and F. Yao, "Exponential stability analysis of impulsive stochastic functional differential systems with delayed impulses," Communications in Nonlinear Science and Numerical Simulation, vol. 19, no. 6, pp. 2104-2114, 2014.

[23] F. Yao, F. Deng, and P. Cheng, "Exponential stability of impulsive stochastic functional differential systems with delayed impulses," Abstract and Applied Analysis, vol. 2013, Article ID 548712, 8 pages, 2013.

[24] P. Cheng, F. Deng, and Y. Peng, "Robust exponential stability and delayed-state-feedback stabilization of uncertain impulsive stochastic systems with time-varying delay," Communications in Nonlinear Science and Numerical Simulation, vol. 17, no. 12, pp. 4740-4752, 2012.

[25] J. Yang, S. Zhong, and W. Luo, "Mean square stability analysis of impulsive stochastic differential equations with delays," Journal of Computational and Applied Mathematics, vol. 216, no. 2, pp. 474-483, 2008.

[26] S. Peng and B. Jia, "Some criteria on pth moment stability of impulsive stochastic functional differential equations," Statistics \& Probability Letters, vol. 80, no. 13-14, pp. 1085-1092, 2010.

[27] C. Li and J. Sun, "Stability analysis of nonlinear stochastic differential delay systems under impulsive control," Physics Letters A, vol. 374, no. 9, pp. 1154-1158, 2010. 


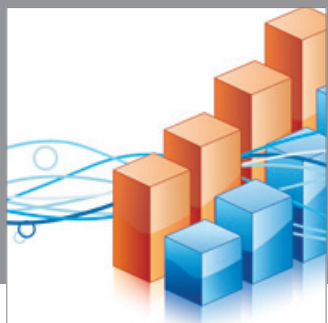

Advances in

Operations Research

mansans

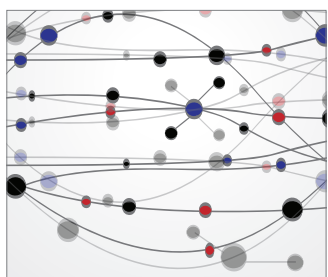

The Scientific World Journal
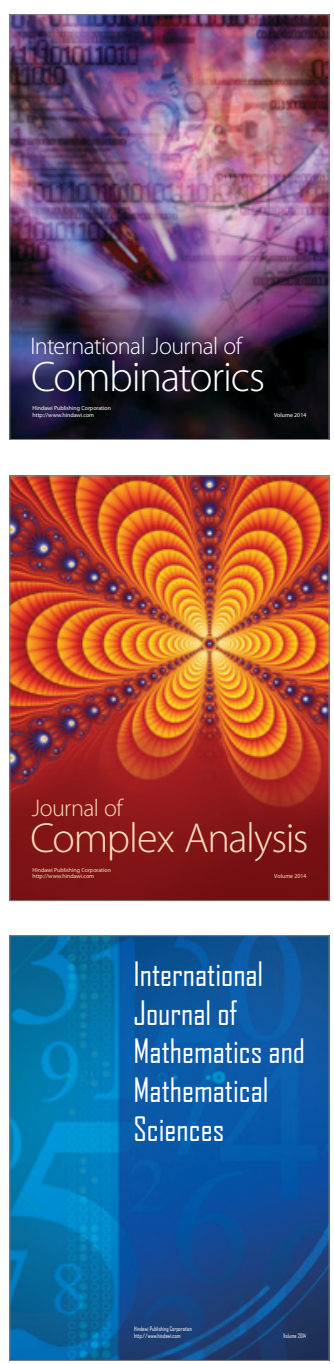
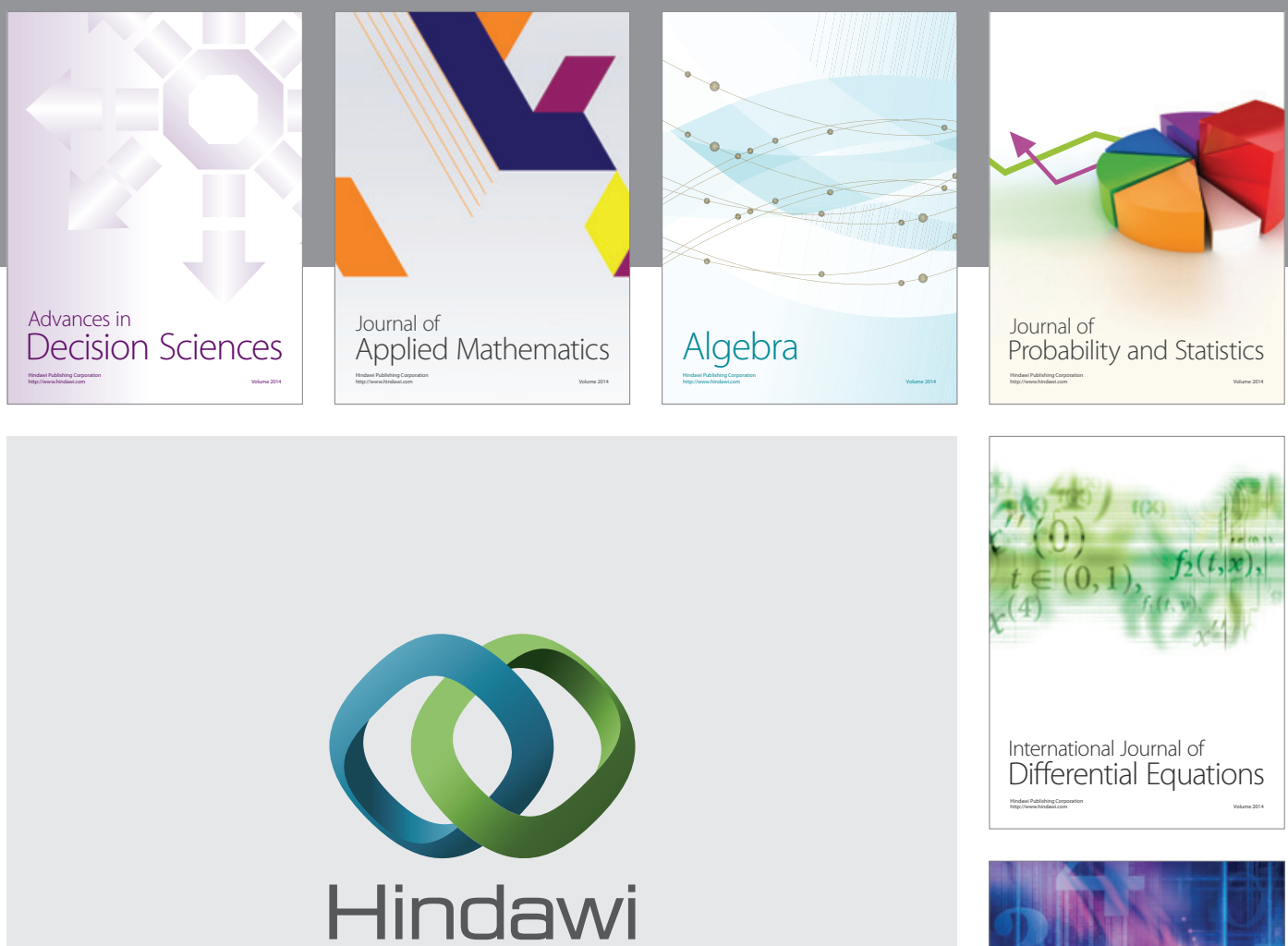

Submit your manuscripts at http://www.hindawi.com
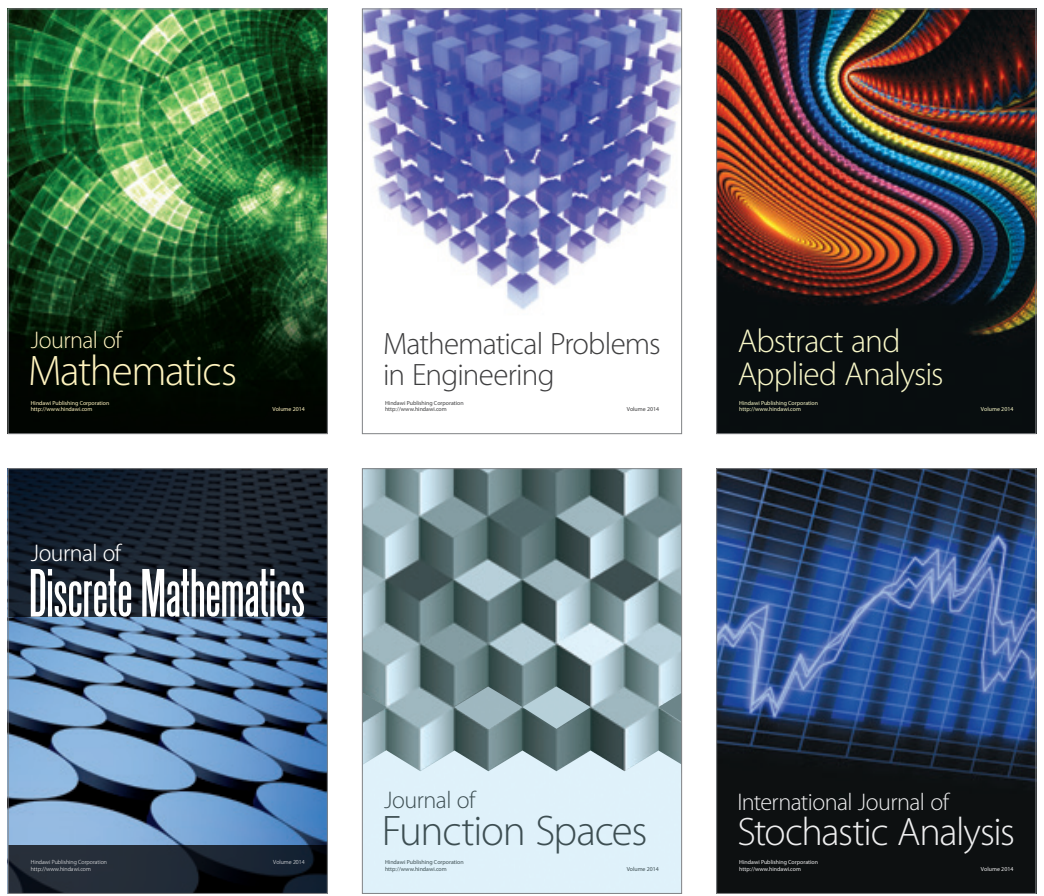

Journal of

Function Spaces

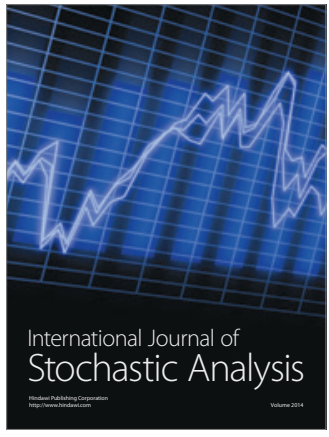

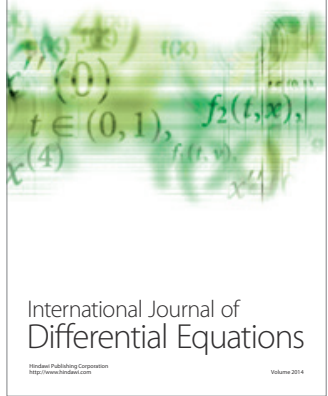
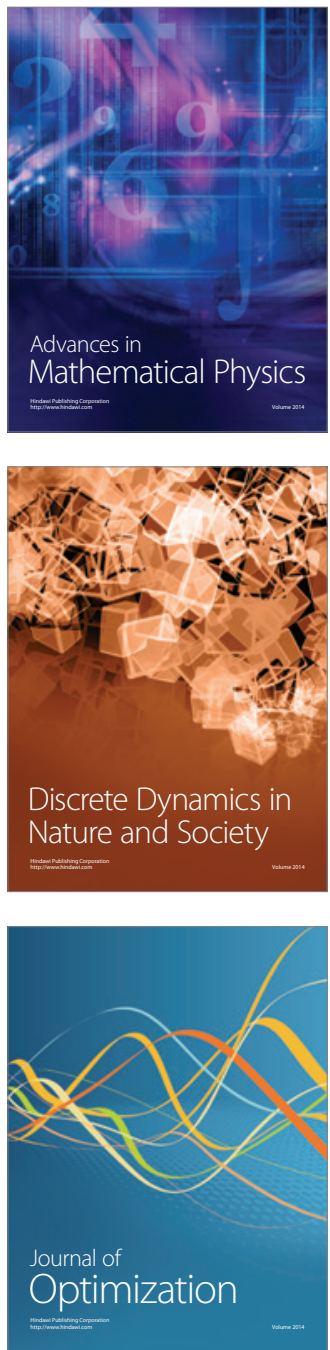